(c) 2009 IEEE. Personal use of this material is permitted. Permission from IEEE must be obtained for all other uses, in any current or future media, including reprinting/republishing this material for advertising or promotional purposes, creating new collective works, for resale or redistribution to servers or lists, or reuse of any copyrighted component of this work in other works. 


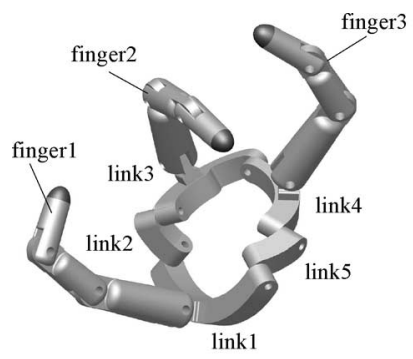

(a)

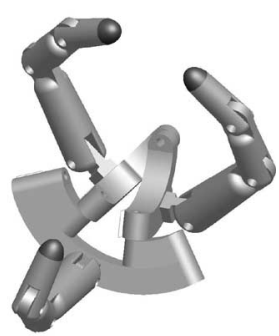

(b)

\section{Orientation and Workspace Analysis of the Multifingered Metamorphic Hand-Metahand}

\author{
Jian S. Dai, Member, IEEE, Delun Wang, and Lei Cui
}

\begin{abstract}
This paper introduces for the first time a metamorphic palm and presents a novel multifingered hand, known as Matahand, with a foldable and flexible palm that makes the hand adaptable and reconfigurable. The orientation and pose of the new robotic hand are enhanced by additional motion of the palm, and workspace of the robotic fingers is complemented with the palm motion. To analyze this enhanced workspace, this paper introduces finger-orientation planes to relate the finger orientation to palm various configurations. Normals of these orientation planes are used to construct a Gauss map. Adding an additional dimension, a 4-D ruled surface is generated to illustrate orientation and pose change of the hand, and an orientation-pose manifold is developed from the orientation-pose ruled surface. The orientation and workspace analysis are further developed by introducing a triangular palm workspace that evolves into a helical surface and is further developed into a 4-D representation. Simulations are presented to illustrate the characteristics of this new dexterous hand.
\end{abstract}

Index Terms-Analysis, dexterous hand, Gauss map, Metahand, metamorphic mechanisms, multifingered hand, orientation, palm, pose, workspace.

\section{INTRODUCTION}

In the past decades, a number of robotic hands have been developed [1], [2]. This has resulted in a great variety of robotic hands. As far as mechanical hands concerned, there are the three-fingered Stanford/JPL hand [3], the four-fingered Utah/MIT hand [4] and Karlsruhe Dexterous II Hand [5], [6], and the five-fingered Belgrade/USC hand [7], DLR hand [8], [9], and NAIST-Hand [10]. These also include the Robonaut hand [11], MANUS-HAND [12], and UBH3 hand [13]. These mechanical hands have played important roles in industrial automation and space technology. In most mechanical hands, fingers are fixed on a rigid palm, and there is no relative lateral movement between fingers, resulting in limited dexterity. This has prompted researchers to look into more dexterous robotic hands. Bonivento et al. [14] proposed a threedegree-of-freedom architecture for a trade-off between a gripper and a multidegree-of-freedom hand to increase fingers' orientation. Baek et al. [15] developed a linkage-driven finger actuated by a single
Fig. 1. Metamorphic hand-Metahand. (a) Spreading palm and (b) folding palm.

motor to imitate human finger motion. The Shadow Robot Company [16] developed an anthropomorphic hand based on pneumatic actuators.

In both mechanical and anthropomorphic hands, a common feature is that a palm is rigid and fixed. The improvement of this resulted in DLR II hand [17] and HIT/DLR hand [18] by breaking a palm into two parts to form relative motion between fingers. In the three-fingered Barrett hand [19] and the underactuated hand generated by Gosselin and Laliberte [20], three fingers can rotate about the central axis at the center of a rigid palm. The design is adopted by [21] and [22]. Higashimori et al. [23] improved the design by proposing a new fourfingered hand with a dual turning mechanism. The four fingers of the new hand are divided into two groups with two fingers in each group that can independently rotate about the central axis.

Though there is relative motion between fingers, a palm of a robotic hand does not have more versatility, which is the main limitation of robotic hands in contrast to a human hand with a foldable and flexile palm. This incurs limitation for manipulation. In particular, conventional design of a finger is to have three parallel joints, and the finger usually operates on a plane. It is then difficult to be adapted to the geometric shape of a manipulated object and to realize fine manipulation. This limits the fine-tuning ability [24] and thus, the use in prosthetics.

This leads to using metamorphic mechanisms as a palm. The metamorphic mechanisms [25], [26] are a class of mechanisms that have the facility to alter a topological configuration from one to another with a resultant change in the mobility of movement. Originating from artworks, this class of mechanisms can be extracted from origami folds that change topological structure [27] during motion.

Applying the principle of metamorphic mechanisms, this paper presents a novel robotic hand, Matahand, by introducing a metamorphic palm that generates reconfigurable motion. The structural change can be used to finely tune the hand pose to grasp an object [28]. Mapping the orientation space of this new hand to an orientation-pose ruled surface, a typical feature of the foldable palm can be presented in its effect on finger motion and workspace of the palm. This mapping reveals the relationship between the orientation-pose space and palm workspace and the performance of the hand and leads to demonstration of the new robotic hand in both virtual environment and demonstrator form.

\section{Metamorphic Multifingered DeXterous Hand AND ORIENTATION-POSE ANALYSIS OF THE METAMORPHIC HAND}

Based on a metamorphic mechanism extracted from an origami fold, a multifingered dexterous hand, Matahand [29], has been developed with this mechanism as its reconfigurable palm in Fig. 1.

This metamorphic hand comprises a specially designed spherical linkage attached with fingers. The base link is link 1 that finger 1 is mounted. There are two cranks in both sides of the base link with 


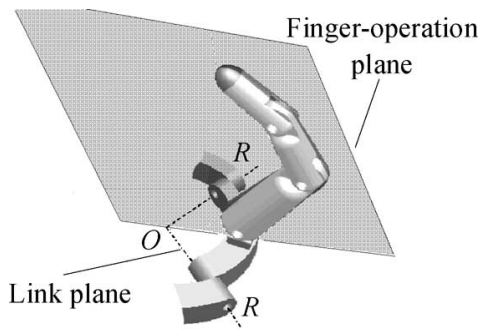

Fig. 2. Finger-operation plane.

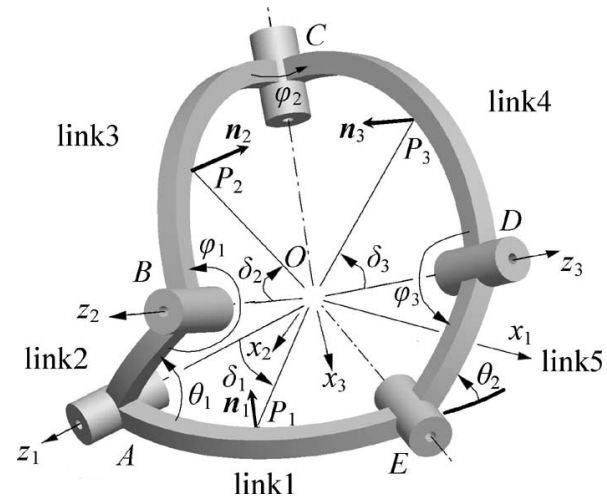

Fig. 3. Palm parameters.

its left-hand-side connecting the first crank link 2 and the right-handside connecting the second crank link 5 . Going clockwise, the link connecting the first crank link is link 3 that mounts finger 2, and the next link is link 4 that mounts finger 3 . The length of links $1-5$ is $l_{1}-l_{5}$.

The palm is foldable and flexible and operates with the degrees of freedom (DOF). Its operation varies the configuration that subsequently changes the finger base positions and finger orientation and, consequently, the hand orientation and pose to adapt to different tasks, including pinching and twisting. This versatile palm makes the hand close to a human hand with a foldable palm and increases the adaptability of the hand.

Since the fingers are based on a foldable palm, finger motion needs to be integrated with the palm motion. This integration is new due to the particular characteristics of this type of robotic hands and a virtual plane needs to be introduced to identify the fingers' orientation associated with the palm motion.

Consider each finger consisting of three revolute joints and operating on a virtual plane perpendicular to a spherical link of the palm where the finger is mounted, the plane is used to define the finger orientation and present the finger axial orientation and lateral orientation by integrating it to the palm motion while it folds and flexes. This plane is named as a finger-orientation plane, which is perpendicular to the link plane defined by the palm link where the finger is mounted, as in Fig. 2.

The schematic installation of fingers is shown in Fig. 3, where finger 1 is fixed at point $P_{1}$ of link 1 , finger 2 at point $P_{2}$ of link 3 , and finger
3 at point $P_{3}$ of link 4 . Since the palm operates in a sphere, each link of the palm is an arc in the sphere, and two input angles $\theta_{1}$ and $\theta_{2}$ are provided by two crank links 2 and 5 . Angles $\varphi_{1}, \varphi_{2}$, and $\varphi_{3}$ are three passive variables and are functions of $\theta_{1}$ and $\theta_{2}$ as in Fig. 3 .

Three normals are required to be obtained to set up the three finger-operation planes by setting three local coordinate frames $O x_{1} y_{1} z_{1}, O x_{2} y_{2} z_{2}$, and $O x_{3} y_{3} z_{3}$ with respect to links 1,3 , and 4, respectively, where $z_{i}$ is the axis of the revolute joint originated from the center of the sphere, and $x_{i}$ lies in the link plane as in Fig. 2 and is perpendicular to $z_{i}$. Since each finger-operation plane is perpendicular to the link plane formed by the palm link and the joint axis, the vector $\boldsymbol{n}_{i}$, which is perpendicular to $O P_{i}$, represents the normal of the corresponding finger-operation plane. Thus, three normals $\boldsymbol{n}_{i}$ can be presented in the respective local coordinate frame as

$$
\begin{aligned}
{ }^{1} \boldsymbol{n}_{1} & =\left[\begin{array}{lll}
c \delta_{1} & 0 & -s \delta_{1}
\end{array}\right]^{\mathrm{T}} \\
{ }^{2} \boldsymbol{n}_{2} & =\left[\begin{array}{lll}
-c \delta_{2} & 0 & -s \delta_{2}
\end{array}\right]^{\mathrm{T}} \\
{ }^{3} \boldsymbol{n}_{3} & =\left[\begin{array}{lll}
-c \delta_{3} & 0 & -s \delta_{3}
\end{array}\right]^{\mathrm{T}}
\end{aligned}
$$

where $c$ and $s$ represent cosine and sine, respectively, and the presuperscripts indicate local frames $O x_{1} y_{1} z_{1}, O x_{2} y_{2} z_{2}$, and $O x_{3} y_{3} z_{3}$. Fixing the global coordinate frame as $O x_{1} y_{1} z_{1}$ and transforming nor$\mathrm{mal}^{2} \boldsymbol{n}_{2}$ from the local coordinate frame to the global coordinate frame by rotating about $z_{2}$ by angle $\left(-\varphi_{1}\right)$ then about $y_{2}$ by angle $\left(-l_{2}\right)$ followed by rotating about $z_{2}$ by angle $\left(-\theta_{1}\right)$, normal $\boldsymbol{n}_{2}$ can be presented in the global coordinate as

$$
\begin{aligned}
\boldsymbol{n}_{2}= & \mathbf{R}\left(\boldsymbol{z}_{2}, \theta_{1}\right) \mathbf{R}\left(\boldsymbol{y}_{2}, l_{2}\right) \mathbf{R}\left(\boldsymbol{z}_{2}, \varphi_{1}\right)^{2} \boldsymbol{n}_{2} \\
& =\left[\begin{array}{c}
-\left(c \theta_{1} c l_{2} c \varphi_{1}-s \theta_{1} s \varphi_{1}\right) c \delta_{2}-c \theta_{1} s l_{2} s \delta_{2} \\
-\left(s \theta_{1} c l_{2} c \varphi_{1}+c \theta_{1} s \varphi_{1}\right) c \delta_{2}-s \theta_{1} s l_{2} s \delta_{2} \\
s l_{2} c \varphi_{1} c \delta_{2}-c l_{2} s \delta_{2}
\end{array}\right] .
\end{aligned}
$$

Similarly ${ }^{3} \boldsymbol{n}_{3}$ can be transformed to the global coordinate frame by rotating about $z_{3}$ by angle $\left(\varphi_{3}\right)$ and then about $y_{3}$ by angle $\left(-l_{5}\right)$. This is followed by rotating about $z_{3}$ by angle $\left(-\theta_{2}\right)$ and about $y_{3}$ by angle $\left(-l_{1}\right)$. Thus we obtain (5), shown at the bottom of the page.

With the introduction of the above normals $\boldsymbol{n}_{1}, \boldsymbol{n}_{2}$, and $\boldsymbol{n}_{3}$, the finger orientation that is integrated by the palm motion can be presented by these normals. The normals can be mapped onto a Gauss map as in Fig. 4.

This map integrates the finger motion and hand orientation and pose into palm motion and is a tool to describe the relationship of the three finger-orientation planes, applicable to all the similar type of hands with a foldable palm. To visualize the relevant orientation among the three finger-operation planes determined by the unit normal vectors, input variable $\theta_{2}$ of the palm is fixed that the metamorphic palm evolves into a spherical four-bar linkage and another input variable $\theta_{1}$ varies. This is implemented by setting $\theta_{2}$ at a fixed value and varying $\theta_{1}$ from 0 to $2 \pi$. Each value of $\theta_{1}$ yields a new configuration of the three finger-orientation planes. Studying the corresponding normals $\boldsymbol{n}_{1}, \boldsymbol{n}_{2}$, and $\boldsymbol{n}_{3}$ with respect to input $\theta_{1}$, orientation of normal

$$
\begin{aligned}
\boldsymbol{n}_{3}= & \mathbf{R}\left(\boldsymbol{y}_{3}, l_{1}\right) \mathbf{R}\left(\boldsymbol{z}_{3}, \theta_{2}\right) \mathbf{R}\left(\boldsymbol{y}_{3}, l_{5}\right) \mathbf{R}\left(\boldsymbol{z}_{3},-\varphi_{3}\right)^{3} \boldsymbol{n}_{3} \\
= & {\left[\begin{array}{c}
\left(\left(s l_{1} s l_{5}-c l_{1} c \theta_{2} c l_{5}\right) c \varphi_{3}-c l_{1} s \theta_{2} s \varphi_{3}\right) c \delta_{3}-\left(c l_{1} c \theta_{2} s l_{5}+s l_{1} c l_{5}\right) s \delta_{3} \\
-\left(s \theta_{2} c l_{5} c \varphi_{3}-c \theta_{2} s \varphi_{3}\right) c \delta_{3}-s \theta_{2} s l_{5} s \delta_{3} \\
\left(\left(s l_{1} s l_{5}-s l_{1} c \theta_{2} c l_{5}\right) c \varphi_{3}+s l_{1} s \theta_{2} s \varphi_{3}\right) c \delta_{3}-\left(c l_{1} c l_{5}-s l_{1} c \theta_{2} s l_{5}\right) s \delta_{3}
\end{array}\right] }
\end{aligned}
$$




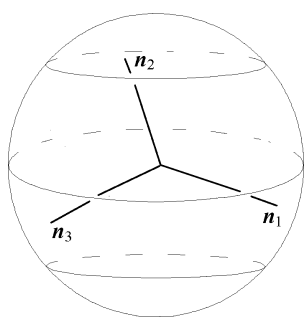

Fig. 4. Gauss map of $\boldsymbol{n}_{1}, \boldsymbol{n}_{2}$, and $\boldsymbol{n}_{3}$ with one configurations of the palm.

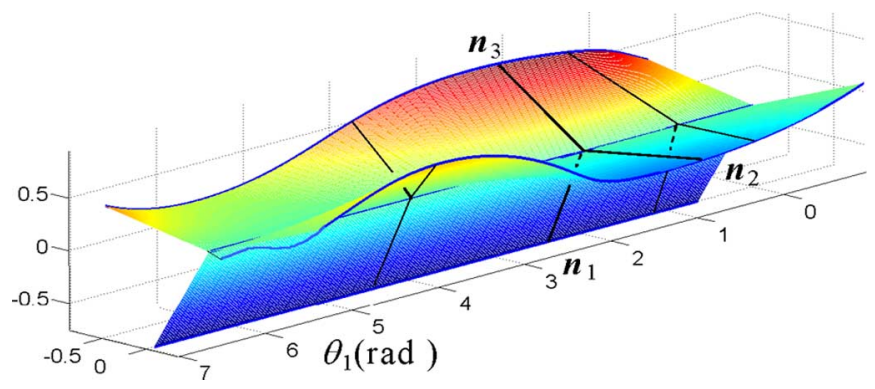

Fig. 5. Orientation-pose ruled surface.

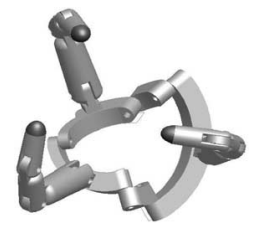

Fig. 6. Corresponding configuration and pose of the metamorphic hand.

$\boldsymbol{n}_{1}$ remains unchanged, and orientation of normals $\boldsymbol{n}_{2}$ and $\boldsymbol{n}_{3}$ vary following the change of fingers 2 and 3 . Thus, the orientation relationship between normals $\boldsymbol{n}_{1}, \boldsymbol{n}_{2}$, and $\boldsymbol{n}_{3}$ varies with the input variable. Map this relationship onto a $3-\mathrm{D}$ space and introduce the forth dimension as input angle $\theta_{1}$ in $x$-axis, $\theta_{1}$, normal $\boldsymbol{n}_{1}$ generates a plane and normals $\boldsymbol{n}_{2}$ and $\boldsymbol{n}_{3}$ generate two ruled surfaces. This gives the orientation-pose ruled surface in Fig. 5.

Corresponding to the marked set of normals $\boldsymbol{n}_{1}, \boldsymbol{n}_{2}$, and $\boldsymbol{n}_{3}$ as in Figs. 4 and 5, the configuration and pose of the metamorphic hand is given in Fig. 6.

When another input variable $\theta_{2}$ of the palm varies, the metamorphic palm has 2 DOF to reconfigure the palm and thus alter the hand orientation and pose. This is represented with a group of the orientation-pose ruled surfaces, which can be used to obtain the values of the two input variables from the palm and the Metahand to adapt to objects with different geometric shapes.

\section{WORKSPACE ANALYSIS OF THE METAMORPHIC HAND}

In the metamorphic hand, the palm motion enhances finger orientation and workspace and subsequently the hand orientation and pose. The palm configuration can be represented by a triangle determined by finger mounting points $P_{1}, P_{2}$, and $P_{3}$ in Fig. 7(a) and a Gauss map in Fig. 7(b).

This triangle varies with the palm motion and represents palm workspace. The expression of $O P_{i}$ in the global coordinate system can be obtained in a similar way to that of $\boldsymbol{n}_{i}$.

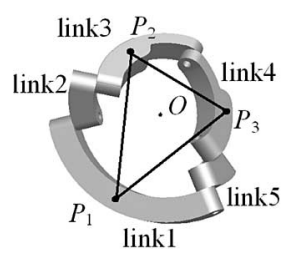

(a)

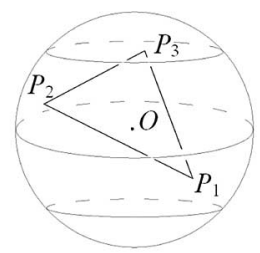

(b)
Fig. 7. Workspace triangle of the metamorphic palm. (a) A workspace triangle and (b) Gauss map of the workspace triangle.

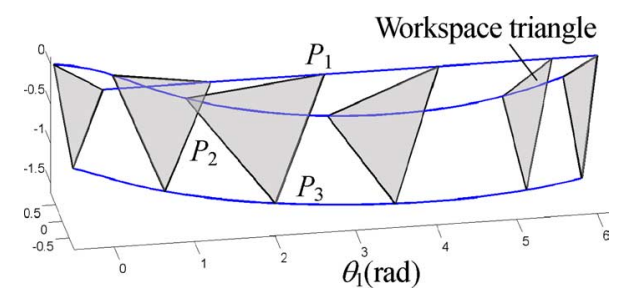

Fig. 8. Workspace helical tube.

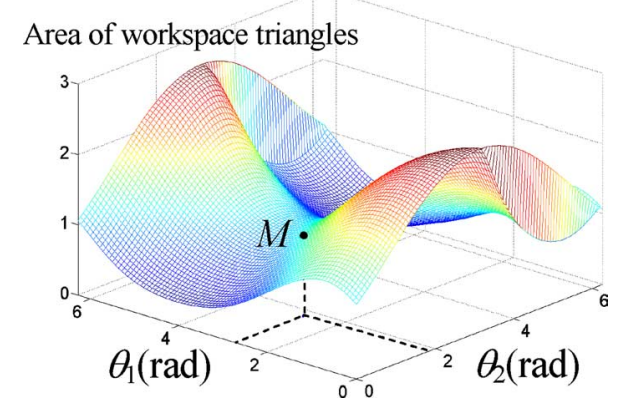

Fig. 9. Values of areas of the workspace triangles in joint space.

Similar to generating the orientation-pose ruled surface in Section II, the workspace triangles are mapped onto a 4-D space with input angle $\theta_{1}$ as an additional dimension along the $x$-axis in Fig. 8. This gives a helical sweep of the workspace triangles, forming the workspace helical tube in the figure.

From the workspace helical tube, the variation of input angle $\theta_{1}$ at the palm gives the configuration variation of the palm and subsequently results in the change of finger orientation and hand pose.

For a given set of input variables $\theta_{1}$ and $\theta_{2}$, the area of the corresponding triangle $P_{1} P_{2} P_{3}$ can be computed. Varying both variables $\theta_{1}$ and $\theta_{2}$, the areas of the series of corresponding palm workspace triangle can be plotted in Fig. 9. The bigger the area is, the larger the workspace will be. In Fig. 9, two horizontal axes represent the input angles $\theta_{1}$ and $\theta_{2}$.

The value of the point $M$ given in Fig. 9 is the area of the marked triangle $P_{1} P_{2} P_{3}$ in Figs. 7 and 8 . For a rigid-palm robotic hand, the palm workspace reduces to a point to an origin of the hand workspace. With the new development, the palm workspace is a polygonal structure. The hand workspace is built on this palm workspace. This enhances and enlarges the hand workspace that generates various hand configurations and poses. 


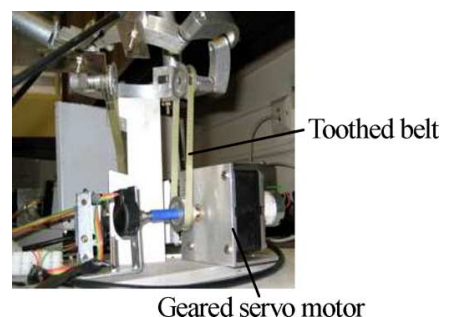

Fig. 10. Actuation system of the palm.
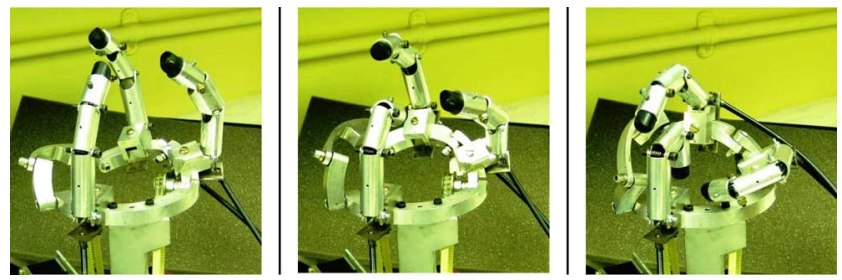

Fig. 11. Motion of the palm and the corresponding poses of the Metahand.

\section{FunCtionality STUdy With THE DEMONSTRATOR AND IN AN VIRTUAL ENVIRONMENT}

Functionality of the metamorphic hand, Matahand, was investigated with a demonstrator and in a virtual environment. The mechanical design started from the palm. This constitutes the orientation and workspace analysis of the hand. The actuation of the palm and the three fingers is implemented by five geared servomotors at a speed range of up to $344 \mathrm{r} / \mathrm{min}$ with a maximal torque of $5 \mathrm{Nm}$ and a gear reducer ratio of 12.5. Each of the three fingers is driven by a motor and is thus underactuated. The remaining two motors are connected adjacent to both sides of the base link of the palm to operate the foldable palm through toothed belt as in Fig. 10. Each servomotor is fixed on the base and to be installed in the arm rather than directly in the palm to reduce the inertial effect.

A series of frames are shown in Fig. 11 that depicts the motion of the palm and the orientation and pose of the Metahand from spreading to folding.

The foldable palm adapts a robotic hand to objects of various geometric shapes, when the relative orientation of the three fingers varies with the palm motion. The three configurations and poses of the metamorphic hand in Fig. 12 are used to grasp objects of different geometric features based on Napier's classification of hand grasps [30].

It can be seen that the foldable and deployable palm adds dexterity to a robotic hand to form various orientation and poses. The workspace of the hand is enhanced by the palm workspace. A larger palm workspace enables a grasped object to have a larger range of pose change. This can be readily seen in Fig. 13(a), where the palm workspace reaches its maximum, and in Fig. 13(b), where the workspace reaches its minimum.

Further to the aforementioned features and different from other robotic hands, the metamorphic hand, Metahand, has features of collapsibility and deployability when implementing a grasp for a pinhole operation and rescue work. The unique movement is illustrated in Fig. 14 as the hand redeploys itself after going through a hole in a collapsible configuration.

A further unique feature of the Metahand is twisting motion that cannot be achieved by other robotic hands since it involves both lateral and radial movements of fingers being substantiated by this foldable and deployable palm. This contrasts with other rotatable palms that
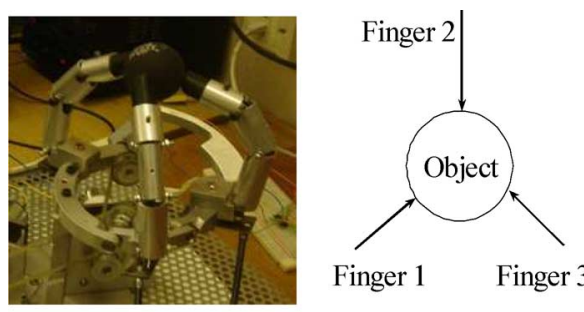

Finger $1 \quad$ Finger 3

(a)
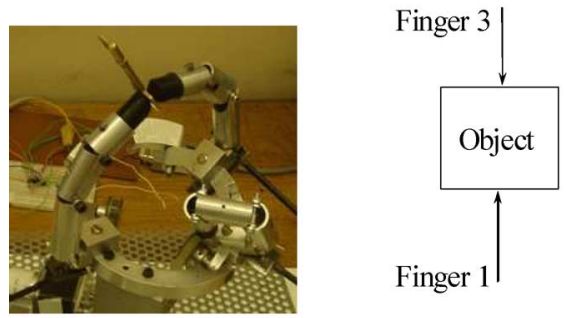

(b)
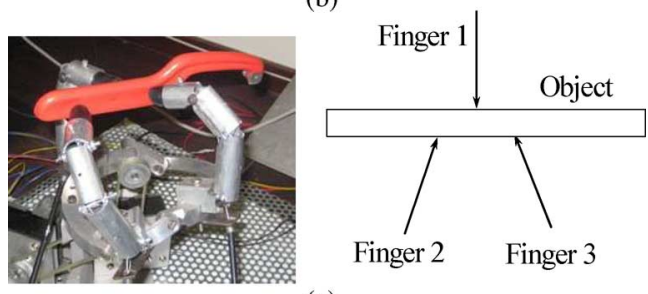

Finger 2 Finger 3

(c)

Fig. 12. Metahand adapting for objects with different geometric shapes (a) Grasp/pinch, (b) snap, and (c) palmer.

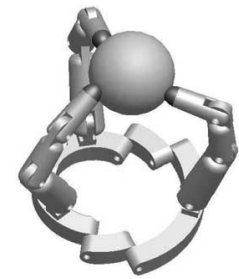

(a)

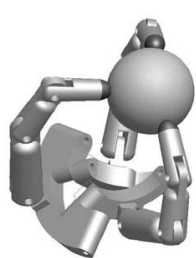

(b)
Fig. 13. Palm workspaces and the corresponding hand poses. (a) Maximum palm workspace and (b) minimum palm workspace.

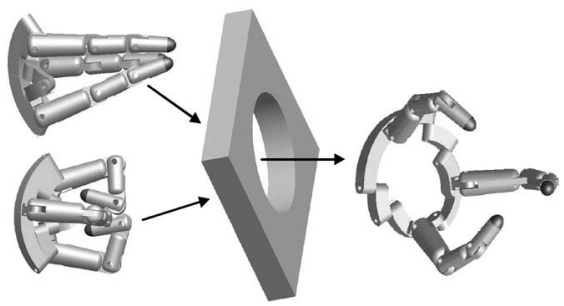

Fig. 14. Folding and redeploying of the Metahand.

make the fingers radially directed. Demonstrated in Fig. 15, a twisting motion is illustrated by rotating the first crank link 2 by $\theta_{1}$ of $23^{\circ}$ with respect to base link 1 and by rotating the second crank link 5 by $\theta_{2}$ of $10^{\circ}$ with respect to the base link.

These inputs make finger 2 move away from the intersecting point, resulting in clockwise rotation, and finger 3 moving away from the intersecting point toward a tangential direction of the ball contributing to the clockwise rotation. This induces twisting motion. 


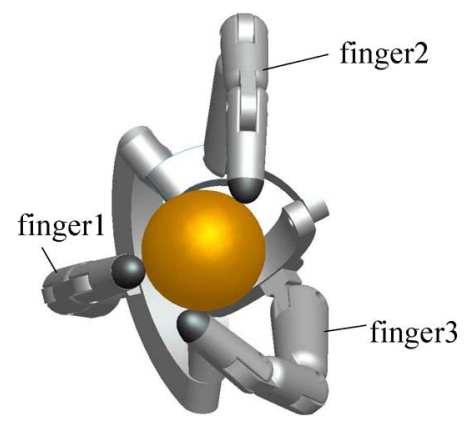

Fig. 15. Twisting motion.

\section{CONCLUSION}

Inspired by origami and artworks and as a philosophy of art-mimetics [26], [38], [39], this paper introduced a new robotic hand, Metahand, which has a foldable and deployable palm. The introduction of this metamorphic palm adds dexterity and versatility to a robotic hand.

To explain the hand orientation and pose that are enhanced by the palm motion, the finger-orientation planes were used to resolve the integration of the finger motion and palm motion. The planes were further presented with their normals in a Gauss map. Relating the relevant motion of finger-orientation planes to the palm variables, the Gauss map is extended to a 4-D manifold that hand orientation and pose and palm configurations can be identified. The fine motion is thus achieved by changing the input palm variables. The paper further verified the motion based on Napier's classification of human hand's grasps and presented typical features of collapsible and twisting motion of the metamorphic hand, Metahand.

\section{REFERENCES}

[1] A. M. Okamura, A. Smaby, and M. R. Cutkosky, "An overview of dexterous manipulation," in Proc. IEEE Int. Conf. Robot. Autom., 2000, pp. 255262.

[2] D. Alba, M. Armada, and R. Ponticelli, "An introductory revision to humanoid robot hands," presented at the 7th Int. Conf. Climbing Walking Robots, Madrid, Spain, Sep. 22-24, 2004.

[3] J. K. Salisbury and J. J. Craig, "Articulated hands: Force control and kinematic issues," Int. J. Robot. Res., vol. 1, no. 1, pp. 4-17, 1982

[4] S. C. Jacobasen, E. K. Iversen, D. F. Knutti, R. T. Johnson, and K. B. Biggers, "Design of the Utah/M.I.T. dexterous hand," in Proc. IEEE Int. Conf. Robot. Autom., San Francisco, CA, 1986, pp. 1520-1532.

[5] D. Osswald and H. Wörn, "Karlsruhe II: Mechanical system and control system of a dexterous robot hand," in Proc. IEEE-RAS Int. Conf. Humanoid Robots, 2001, pp. 407-414.

[6] N. Fukaya, S. Toyama, T. Asfour, and R. Dillmann, "Design of the TUAT/Karlsruhe humanoid hand," in Proc. IEEE/Robot.-Soc.-of-Jpn. Int. Conf. Intell. Robots Syst., Kagawa Univ., Takamatsu, Japan, Oct. 31-Nov. 5, 2000, vol. 1-3, pp. 1754-1759.

[7] R. Tomovic, G. A. Berkey, and W. J. Karplus, "A strategy for grasp synthesis with multifingered robot hand," in Proc. IEEE Int. Conf. Robot. Autom., Raleigh, NC, 1987, pp. 83-89.

[8] J. Dietrich, G. Hirzinger, J. Heindl, and J. Scott, "Multisensory telerobotic techniques," in Traditional and Non-Traditional Robotic Sensors. Berlin, Germany: Springer-Verlag, 1999.

[9] G. Hirzinger, K. Landzettel, B. Brunner, I. Schaefer, M. Fischer, M. Grebenstein, N. Sporer, M. Schott, M. Schedl, and C. Deutrich, "DLR's robotics lab-Recent developments in space robotics," in Proc. 5th Symp. Artif. Intell., Robot. Autom. Space, Noordwijk, Netherlands, Jun. 1-3, 1999, pp. 25-34.

[10] J. Ueda, Y. Ishida, M. Kondo, and T. Ogasawara, "Development of the NAIST-hand with vision-based tactile fingertip sensor," in Proc. IEEE Int. Conf. Robot. Autom., 2005, vol. 2, pp. 2332-2337.

[11] C. Lovchik and M. Diftler, "The robonaut hand: A dexterous robot hand for space," in Proc. IEEE Int. Conf. Autom. Robot., Detroit, MI, 1999, vol. 2, pp. 907-912.
[12] J. L. Pons, E. Rocon, R. Ceres, D. Reynaerts, B. Saro, S. Levin, and W. Van Moorleghem, "The MANUS-HAND dexterous robotics upper limb prosthesis mechanical and manipulation aspects," in Autonomous Robots, vol. 16, Norwell, MA: Kluwer, 2004, no. 2, pp. 143-163.

[13] F. Lotti, P. Tiezzi, and G. Vassura, "UBH3: Investigating alternative design concepts for robotic hands," in Proc. IEEE Int. Conf. Robot. Autom., 2004, vol. 5, p. 4736.

[14] C. Bonivento, C. Melchiorri, G. Vassura, G. Ferretti, C. Maffezzoni, G. Magnani, G. Beccari, S. Caselli, and F. Zanichelli, "A dexterous gripper for space robotics," in Proc. 5th Int. Symp. Artif. Intell., Robot. Autom. Space, Noordwijk, Netherlands, Jun. 1-3, 1999, vol. 440, pp. 637-642.

[15] S. E. Baek, S. H. Lee, and J. H. Chang, "Development of a robotic finger for prosthetic hands," in Proc. 7th Int. Conf. Rehabil. Robot., Integr. Assistive Technol. Inform. Age, Stanford Univ., Stanford, CA, 2001, vol. 9, pp. 9-15.

[16] Shadow Robot Co., "Developments in dexterous hands for advanced robotic applications," in Proc. 10th Int. Symp. Robot. Appl., Seville, Spain, Jun. 28-Jul. 1, 2004, vol. 15, pp. 123-128.

[17] J. Butterfass, M. Grebenstein, and H. Liu, "DLR-Hand II: Next generation of a dexterous robot hand," in Proc. IEEE ICRA, Seoul, Korea, 2001, pp. 413-428.

[18] X. Gao, S. Shi, D. Zhao, L. Jiang, Z. Wang, H. Cai, and H. Liu, "HIT anthropomorphic robotic hand and finger motion control," J. Harbin Inst. Technol. (New Series), vol. 13, no. 1, pp. 49-51, 2006.

[19] W. T. Townsend, "The BarrettHand grasper-programmably flexible part handling and assembly," Ind. Robot, vol. 27, no. 3, pp. 181-188, 2000.

[20] C. M. Gosselin and T. Laliberte, "Underactuated mechanical finger with return actuation [P]," U.S. Patent 5762 390, 1998.

[21] A. Edsinger-Gonzales. (2005). Design of a compliant and force sensing hand for a humanoid robot [Online]. Available: http://people.csail. mit.edu/edsinger/doc/edsinger_img04.pdf

[22] X. T. Le, W. G. Kim, B. C. Kim, S. H. Han, J. G. Ann, and Y. H. Ha, "Design of a flexible multifingered robotics hand with 12 D.O.F. and Its control applications," presented at the SICE-ICASE Int. Joint Conf., Busan, Korea, Oct. 18-21, 2006.

[23] M. Higashimori, H. Jeong, I. Ishii, M. Kaneko, A. Namiki, and M. Ishikawa, "A new four-fingered robot hand with dual turning mechanism," in Proc. 2005 IEEE Int. Conf. Robot. Autom. Barcelona, Spain, Apr., pp. 2679-2684.

[24] A. Ghafoor, J. S. Dai, and J. Duffy, "Fine motion control based on constraint criteria under pre-loading configurations," J. Robot. Syst., vol. 17, no. 4, pp. 171-185, 2000.

[25] J. S. Dai and J. R. Jones, "Mobility in metamorphic mechanism of foldable/erectable kinds," presented at the 25th ASME Biennial Mechanisms Robot. Conf., Amer. Soc. Mech. Eng., Baltimore, MD, 1998.

[26] J. S. Dai and J. R. Jones, "Mobility in metamorphic mechanism of foldable/erectable kinds," ASME Trans., J. Mech. Design, vol. 121, no. 3, pp. 375-382, 1999.

[27] J. S. Dai and J. R. Jones, "Matrix representation of topological changes in metamorphic mechanisms," ASME Trans., J. Mech. Design, vol. 127, no. 4, pp. 675-682, 2005.

[28] A. Ghafoor, J. S. Dai, and J. Duffy, "Stiffness modeling of a soft-finger contact in robotic grasping," Trans. ASME: J. Mech. Design, vol. 126, no. 4, pp. 646-656, 2004.

[29] J. S. Dai, "Robotic hand with palm section comprising several parts able to move relative to each other," Patent WO/2005/105391, International Patent PCT/GB2005/001665, U.K. Patent GB04 095 48.5, 2004.

[30] J. Napier, "The prehensile movements of the human hands," J. Bone Joint Surg., vol. 38B, no. 4, pp. 902-913, Nov. 1956.

[31] M. T. Mason and J. K. Salisbury, Robot Hands and the Mechanics of Manipulation. Cambridge, MA: MIT Press, 1985.

[32] Y. L. Xiong, D. J. Sanger, and D. R. Kerr, "Geometric modeling of bounded and frictional grasps," Robotica, vol. 11, pp. 185-192, 1993.

[33] T. Watanabe and T. Yoshikawa, "Grasping optimization using a required external force set," IEEE Trans. Autom. Sci. Eng., vol. 4, no. 1, pp. 52-66, Jan. 2007.

[34] G. Liu, J. Xu, X. Wang, and Z. Li, "On quality functions for grasp synthesis, fixture planning, and coordinated manipulation," IEEE Trans. Autom. Sci. Eng., vol. 1, no. 2, pp. 146-162, Oct. 2004.

[35] R. Platt, Jr., A. H. Fagg, and R. A. Grupen, "Extending fingertip grasping to whole body grasping," in Proc. IEEE Int. Conf. Robot. Autom., Taipei, Taiwan, Sep. 14-19, 2003, pp. 2677-2682.

[36] X. Zhu, H. Ding, and J. Wang, "Grasp analysis and synthesis based on a new quantitative measure," IEEE Trans. Robot. Autom., vol. 19, no. 6, pp. 942-953, Dec. 2003. 
[37] S. Ekvall and D. Kragic, "Learning and evaluation of the approach vector for automatic grasp generation and planning," in Proc. IEEE Int. Conf. Robot. Autom., Roma, Italy, Apr. 10-14, 2007, pp. 4715-4720.

[38] W. Yao and J. S. Dai, "Dexterous manipulation of origami cartons with robotic fingers based on the interactive configuration space," ASME Trans.: J. Mech. Design, vol. 130, no. 2, pp. 022303-1-022303-8, 2008.

[39] E. Rodriguez-Leal and J. S. Dai, "From Origami to a new class of centralized 3-DOF parallel mechanisms, DETC2007-35516," presented at the 31st ASME Mechanisms Robot. Conf., ASME Int. Design Eng. Tech. Conf. Comput. Inform. Eng. Conf., Las Vegas, NV, Sep 4-7, 2007. 